\title{
Trypanosoma Brucei Aquaglyceroporins Facilitate the Uptake of Arsenite and Antimonite in a pH Dependent Way
}

\author{
Néstor L. Uzcátegui ${ }^{a, b} \quad$ Katherine Figarella ${ }^{b}$ Bjoern Bassarak ${ }^{c}$ \\ Nestor W. Mezad Rita Mukhopadhyaye, Jose L. Ramirez ${ }^{\mathrm{b}, \mathrm{f}}$ \\ Michael Duszenkoc \\ aInstitute for Anatomy, Central University of Venezuela, 'biotechnology Center, IDEA Foundation, \\ Caracas, Venezuela; ' Interfaculty Institute of Biochemistry, University of Tübingen, Tübingen, Germany; \\ ${ }^{\mathrm{d} G e n o m a ~ L a b . ~ V e n e z u e l a ~ a n d ~ L A B I E M E T, ~ F a c u l t y ~ o f ~ M e d i c i n e, ~ U n i v e r s i t y ~ o f ~ t h e ~ A n d e s, ~ S a n ~ C r i s t o b a l, ~}$ \\ Venezuela; 'Department of Biochemistry and Molecular Biology, Wayne State University, School of \\ Medicine, Detroit, MI, USA; fUnited Nations University Programme UNU-BIOLAC, Idea Foundation, \\ Caracas, Venezuela; 9Present address: Department of Cellular Biology and Pharmacology College of \\ Medicine, Florida International University, Florida, USA
}

\author{
Key Words \\ Aquaporins $•$ Metalloids $・$ Trypanosomes $・ A s($ III) $・ \mathrm{Sb}(\mathrm{III})$
}

\begin{abstract}
Background: Trypanosoma brucei is a primitive parasitic protozoan that thrives in diverse environments such as the midgut of the tsetse fly and the blood of a mammalian host. For an adequate adaptation to these environments, the parasite's aquaglyceroporins play an important role. Methods and Results: In order to test their ability to transport trivalent arsenic and antimony, we expressed the three known Trypanosoma brucei aquaglyceroporins (TbAQPs) in the heterologous systems of yeast null aquaporin mutant and Xenopus laevis oocytes. For both expression systems, we found a $\mathrm{pH}$ dependent intracellular accumulation of $\mathrm{As}$ (III) or $\mathrm{Sb}$ (III) mediated by all of the three TbAQPs, with the exception of TbAQP1-As(III) uptake. Additionally, we observed that Trypanosoma brucei aquaglyceroporins allow the passage of As(III) in both directions. Conclusion: Taken together, these results demonstrated that $T$. brucei aquaglyceroporins can serve as entry routes for $\mathrm{As}$ (III) and $\mathrm{Sb}$ (III) into the parasitic cell, and that this uptake is $\mathrm{pH}$ sensitive. Therefore, aquaporins of protozoan parasites may be considered useful as a vehicle for drug delivery.
\end{abstract}

Copyright (c) 2013 S. Karger AG, Basel

Universidad Central de Venezuela. Paseo Los Ilustres, Ciudad Universitaria Instituto Anatómico,Caracas 1040 (Venezuela)

University of Tuebingen Interfaculty Institute for Biochemistry

Hope-Seyler Str. 4. 72076 Tuebingen (Germany)

E-Mail nestor.uzcategui@ucv.ve and E-Mail michael.duszenko@uni-tuebingen.de 


\section{Introduction}

In tropical and subtropical areas of the world, members of the Trypasomatidae family cause diseases, and thus represent an important cause of human mortality and morbidity [1]. The more important of these diseases are African sleeping sickness, Chagas disease, and leishmaniasis caused by Trypanosoma brucei, Trypanosoma cruzi, and Leishmania, respectively $[1,2]$. Therapeutic options to treat these diseases are remarkably limited and most of them unsatisfactory, as they have severe side effects and show variable efficacy. Nowadays, arsenic and antimony, although toxic compounds, are still the chemical bases for drugs commonly used against Leishmania and T. brucei [1].

Leishmaniasis in particular is treated with drugs containing pentavalent antimony $[\mathrm{Sb}(\mathrm{V})]$ (Pentostam and Glucantime) whose active form is antimonite [Sb(III)]. For human African sleeping sickness there are few drugs available and out these, eflornithine in combination with nifurtimox, is an effective therapy. However, this treatment, in some cases, has a reduced efficacy due to the weak effect of eflornithine against Trypanosoma brucei rhodesiense [1,3]. Melarsoprol (Arsobal@, Aventis), a highly toxic trivalent organic derivative of arsenic, is one of the few drugs which is effective against both species of Trypanosoma brucei (T. b. rhodesiense and T. b. gambiense), and particularly effective against the chronic stages of the disease [4].

Interestingly, membrane proteins belonging to the aquaporin family, i.e. channels that facilitate the transport of water (orthodox aquaporin), and small non-charged solutes including glycerol (aquaglyceroporins) have been shown to modulate the activity of drugs whose active ingredients are $\mathrm{As}(\mathrm{III})$ or $\mathrm{Sb}(\mathrm{III})$. Leishmania major aquaglyceroporin 1 (LmAQP1) is responsible for $\mathrm{Sb}(\mathrm{III})$ and $\mathrm{As}$ (III) uptake [5], and a down regulation of LmAQP1 is associated with drug resistance [6]. In T. brucei, although it is well known that melarsoprol enters the cell through both the P2 (adenosine transporter) and HAPT1 (high-affinity Pentamidine transporter), aquaglyceroporin 2 plays an important role in mediating the susceptibility to melarsoprol and pentamidine treatment $[3,7,8]$. However, hitherto it was not known whether TbAQPs were permeable to any arsenical compound but considering their pore size, it seems rather unlikely that drugs like melarsoprol could pass through. Although in many organisms As(III) and $\mathrm{Sb}$ (III) are taken up by aquaglyceroporins, it has been clearly demonstrated that not all aquaglyceroporins carry out this function [9]. Here, using two different heterologous expression systems (yeast and Xenopus oocytes), we measured the $\mathrm{Sb}(\mathrm{III})$ and $\mathrm{As}(\mathrm{III})$ facilitated uptake by the TbAQPs, thus providing direct evidence that TbAQPs are arsenic and antimony channels and that the uptake of these metalloids is $\mathrm{pH}$ dependent.

\section{Material and Methods}

\section{Ethics Statement}

This work was performed in accordance with the Guide for the Care and Use of Laboratory Animals recommendations of the National Institutes of Health. The protocol was approved by the Wayne State University Animal Investigation Committee. All efforts were made to minimize suffering; surgery was performed under benzocaine anesthesia, and this compound was also used for animal euthanasia.

\section{Maintenance of organisms}

Saccharomyces cerevisiae: Cells were grown in a yeast peptone medium containing $2 \%$ D-glucose (rich medium) or in a synthetic medium for their selection consisting of complete medium (CM) without uracil (selection marker) [10]. Solid medium was prepared using $2 \%$ agar.

Xenopus laevis oocytes: The oocytes obtained by surgery were defolliculated and manually culled for cells from stages V-VI. Next they were maintained in ND96 solution for $24 \mathrm{~h}$ at $16^{\circ} \mathrm{C}$ with continuous shaking until cRNA was injected [11].

Parasites: T. brucei bloodstream forms were cultivated in modified minimum essential medium at $37^{\circ} \mathrm{C}$ in a $5 \% \mathrm{CO}_{2}$ atmosphere as described previously [12]. 


\section{Heterologous expression in S. cerevisiae and phenotype experiments}

In this study the yeast strain BY4742 (MAT $\alpha$; his3 $\Delta$ 1; leu2 $\Delta$ 0; lys2 $\Delta$ 0; ura3 $\Delta$ 0; YLL043w::kanMX4) from Euroscarf (Frankfurt, Germany) was transformed with the following constructs: pRS416/TbAQP1, pRS416/TbAQP2, and pRS416/TbAQP3 [13]. Expression of TbAQPs was induced by removing methionine from the culture medium (MET17- inducible promoter). BY4742 transformants were grown in selection medium (CM without uracil) at room temperature and shaking at $160 \mathrm{rpm}$ overnight, and then transferred to selection medium plates containing arsenic V, arsenic III, or antimony. Control plates lacked these compounds. Drop tests on agar plates were performed from $1 / 10^{1}$ to $1 / 10^{5}$ dilutions using overnight cultures adjusted to identical cell densities [13].

Transport of arsenic and antimony by TbAQP1, TbAQP2, and TbAQP3 expressed in Xenopus laevis oocytes

For expression in oocytes, $10 \mathrm{ng}$ of cRNA were injected into each oocyte and after three days, when the highest expression level was reached, metalloid uptake assay was performed as described by Figarella et al., 2007 [14]. Briefly, five oocytes were incubated for $90 \mathrm{~s}$ at room temperature with either $1 \mathrm{mM} \mathrm{As(III)}$ or Sb(III) in ND96 buffer. After washing, oocytes were transferred to separate test tubes and digested with concentrated $\left(70 \%\right.$, inductively-coupled plasma (ICP)-GRADE) $\mathrm{HNO}_{3}$ for $1 \mathrm{~h}$ at $70^{\circ} \mathrm{C}$. Each sample was diluted with HPLC grade deionized water to a final concentration of $3 \% \mathrm{HNO}_{3}$ and the metalloid concentration was determined by inductively-coupled plasma mass spectrometry (ICP-MS).

\section{Cytotoxicity assays}

Cytotoxicity was assayed using concentration inhibitory $50\left(\mathrm{IC}_{50}\right.$, the concentration necessary to inhibit cell growth by 50\%). Trypanosoma brucei bloodstream form parasites were collected from in vitro cultures during the exponential growth phase and diluted to $2 \times 10^{5}$ cells per ml, placed in a 96-well plate and grown with or without the following metalloids: As(III) and As(V) in the form of sodium arsenite and arsenate, and $\mathrm{Sb}(\mathrm{III})$ in the form of potassium antimonyl tartrate, at different concentrations. Plates were incubated at $37^{\circ} \mathrm{C}$ in a $5 \% \mathrm{CO}_{2}$ for $24 \mathrm{~h}$. To determine the percentage of dead cells, growth was stopped and phosphatase activity was measured as described by Bodley et al. 1995 [15].

\section{Results}

TbAQPs complement the trivalent arsenic and antimony resistance phenotype in an fps1 strain of S. cerevisiae

Since the Fps1p channel is responsible for trivalent metalloid uptake, yeast strains lacking this gene show a resistant phenotype to $\mathrm{As}(\mathrm{III})$ and $\mathrm{Sb}$ (III) [16]. In order to investigate whether TbAQPs can replace Fps1p in conferring trivalent metalloid sensitivity, we transformed fps $1 \Delta$ mutant cells with each one of the three TbAQPs, or with the vector plasmid (control cells). Transformants were grown in selective medium and then spotted onto agar plates containing serial dilutions of $\mathrm{As}(\mathrm{III}), \mathrm{As}(\mathrm{V})$ or $\mathrm{Sb}(\mathrm{III})$. As shown in Figure 1 , different from control cells, TbAQPs-transformed fps1 $\Delta$ cells were highly sensitive to As(III) and Sb(III). Since the only difference between control cells and TbAQP-transformants is the gene inserted in the vector, we concluded that the enhanced sensitivity to trivalent metalloids is due to the complementation of Fps1p function by TbAQPs, and therefore the metalloid uptake in T. brucei should be mediated by these channels. On the other hand, when TbAQPs-transformed fps1 $1 \Delta$ yeast and control cells were exposed to As (V), the respective phenotypes were inverted, i.e. the transformed cells became resistant and control cells were sensitive. This improved survival of mutants expressing TbAQPs can be explained by the arsenic metabolism in yeast: $\mathrm{As}(\mathrm{V})$ (arsenate) is taken up by the yeast phosphate transporter Pho87p, and once inside the cell, it is reduced to arsenite by the redox enzyme Acr2p, and finally secreted by aquaglyceroporins along the arsenite concentration gradient [17-19]. In contrast, control cells accumulated As(III) and died because they do not have an efficient pathway to get rid of the metalloid. This phenotype confirms and complements the former experiments indicating that TbAQPs were able to participate in the efflux of As(III). 


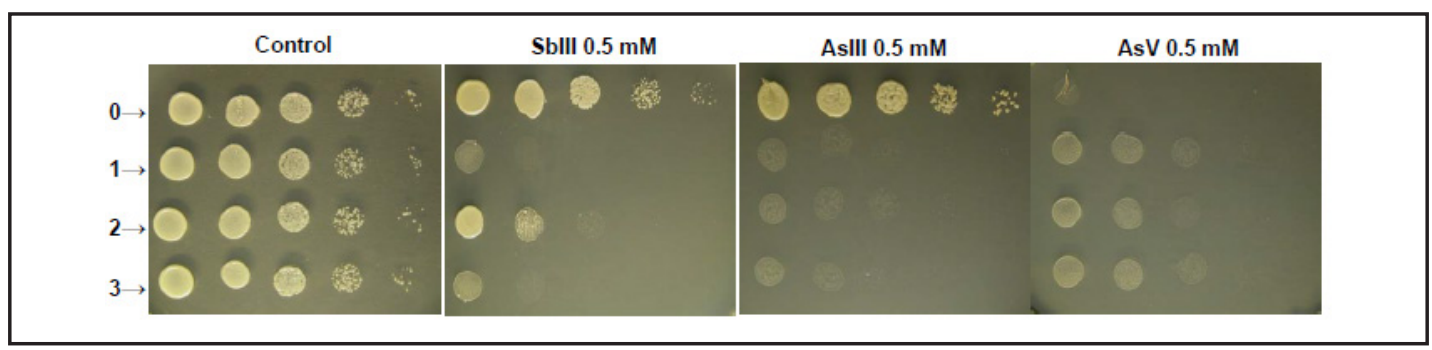

Fig. 1. Phenotypes of fps $1 \Delta$ mutant cells expressing TbAQPs in the presence or absence of metalloids. Yeast cells were pre-grown in selective liquid and the cell density adjusted to 1 O.D. Thereafter, they were transferred in a tenfold serial dilution onto selective-induction agar medium without any metalloids (control condition) or containing $0.5 \mathrm{mM}$ of $\mathrm{Sb}(\mathrm{III})$, As(III), or As(V). Growth was documented after 3 days at $30^{\circ} \mathrm{C}$. In each panel: 0 , control cells containing the empty vector; 1 , TbAQP1; 2, TbAQP2; 3, TbAQP3.

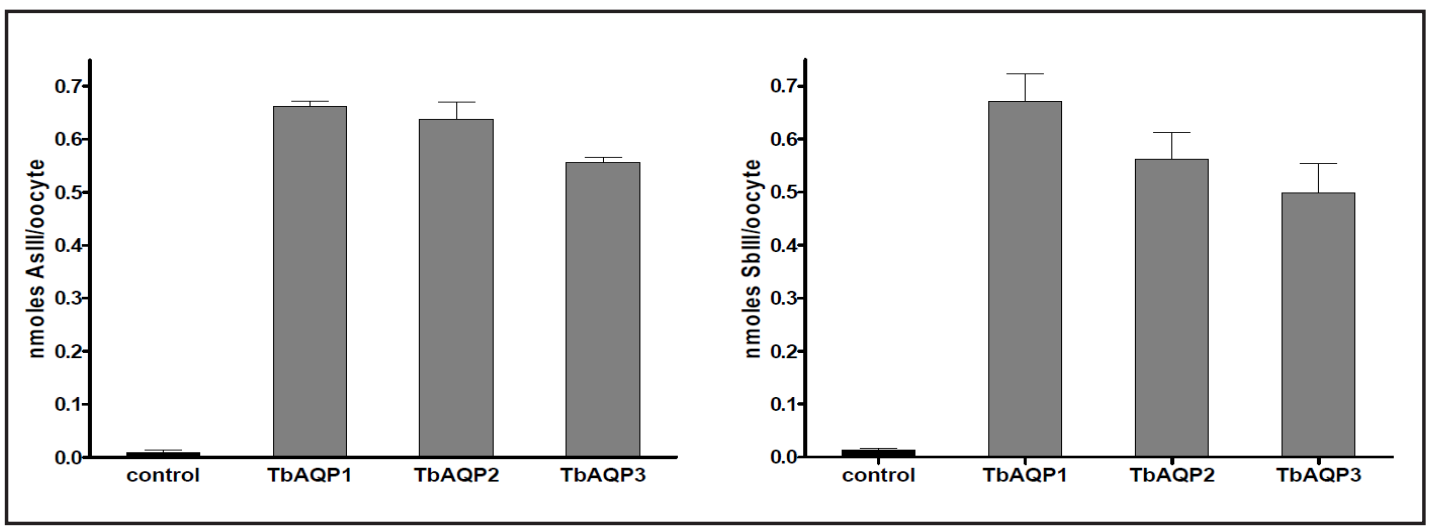

Fig. 2. As(III) and Sb(III) permeability in Xenopus laevis oocytes expressing TbAQP1-3. Oocytes were injected with water (control) (black bar) or 10 ng of TbAQP1, 2 or 3 - cRNA (grey bars). After three days permeability experiments were achieved. Graphics represent measurements performed at least in triplicate \pm SD.

TbAQPs expressed in Xenopus laevis oocytes take up As(III) and Sb(III)

We further characterized As(III) and Sb(III) transport in Xenopus laevis oocytes where recombinant TbAQPs were expressed, by measuring the permeability profiles of these metalloids. Uptake of both As(III) and Sb(III) at a concentration of $1 \mathrm{mM}$ each was monitored in TbAQP1-3 expressing oocytes over a 90 seconds period, the control experiment consisted of water injected oocytes. As shown in Figure 2, control oocytes showed a negligible As(III) or $\mathrm{Sb}$ (III) uptake $(0.006 \pm 0.001 \mathrm{nmol} /$ oocyte and $0.013 \pm 0.008 \mathrm{nmol} /$ oocyte, respectively). On the other hand, oocytes expressing TbAQP 1, 2 or 3 showed a 109-fold, 112-fold, or 91fold accumulation of As(III) respectively when compared with the control. Similar results were obtained for $\mathrm{Sb}(\mathrm{III})$ with a 52 -fold, 46 -fold, or 44 -fold increase. These results clearly demonstrate that the three T. brucei aquaglyceroporins act as metalloid channels.

\section{Effect of $p H$ on metalloids uptake in S. cerevisiae TbAQPs transformants}

Ramírez-Solís et al. have demonstrated that at neutral $\mathrm{pH}$ the most stable structure for $\mathrm{As}(\mathrm{III})$ is $\mathrm{As}(\mathrm{OH})_{3}$, thus apparently, the ionic As atom by itself is not present in solution. However, variations in $\mathrm{pH}$ cause significant structural changes in the coordination geometry of As(III) [20]. Since these changes in spatial geometry could affect the passage of these compounds through the channel's pore, we decided to analyze whether changes in $\mathrm{pH}$ could modify the ability of TbAQPs to transport As(III) and Sb(III). Accordingly, yeast TbAQPtransformants were cultured on agar plates containing As(III) or Sb(III) at different pHs. As shown in Figure 3 in the presence of 0.5 or $1 \mathrm{mM}$ of $\mathrm{Sb}(\mathrm{III})$ the three yeast TbAQPs transformants displayed a moderate growth at $\mathrm{pH}$ of 3.5 , but when the $\mathrm{pH}$ was raised to 
Fig. 3. As(III) and $\mathrm{Sb}(\mathrm{III}) \mathrm{pH}$-dependent cytotoxicity in fps $1 \Delta$ mutant cells expressing TbAQPs. Yeast mutants expressing or not expressing TbAQP1, 2, and 3 were spotted in 10-fold dilution steps onto selectiveinduction agar medium. Media containing As(III) or $\mathrm{Sb}(\mathrm{III})$ was prepared at different $\mathrm{pH}$ from 3.5 to 6.5. Control condition does not have any metalloids. Proliferation was recorded 3 days after incubation at $30^{\circ} \mathrm{C}$. a) Grown in the presence of $\mathrm{Sb}(\mathrm{III})$. b) Grown in the presence of As(III). In each plate: control cells containing an empty vector (0); Cells expressing TbAQP1, TbAQP2, or TbAQP3 (1), (2), or (3), respectively.

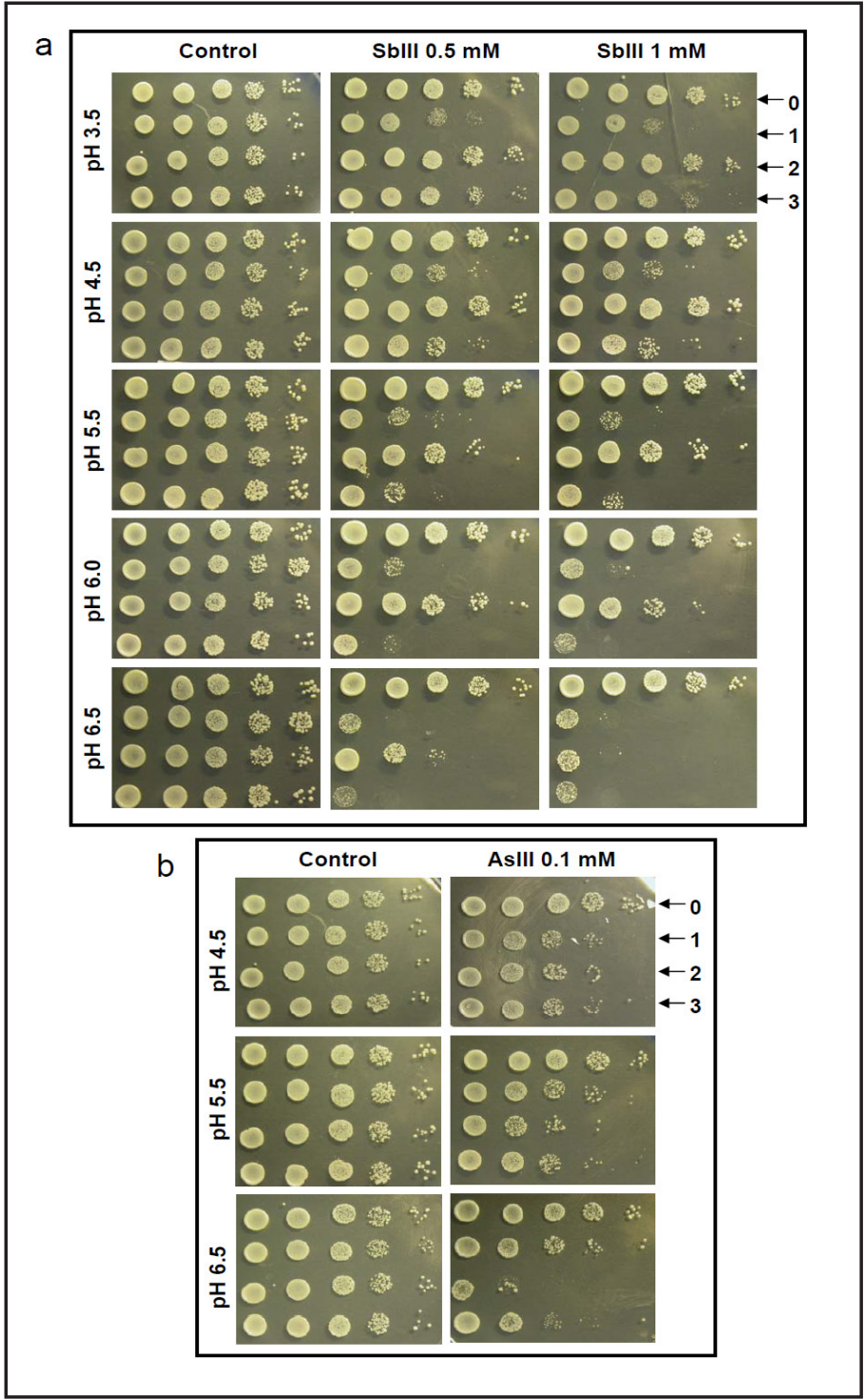

6.5 (Fig. 3a) the sensitivity of these cells to $\mathrm{Sb}$ (III) increased steadily. Similar results were obtained for As(III) in TbAQP2 and 3 transformants but at a lower concentration (Fig. 3b). For TbAQP1-transformed fps $1 \Delta$ mutant cell variations in $\mathrm{pH}$ did not change the transport rate for As(III). In general these results suggest that changes in the spatial structure of $\mathrm{As}(\mathrm{OH})_{3}$ and $\mathrm{Sb}(\mathrm{OH})_{3}$ at low $\mathrm{pH}$ decreased the permeability of TbAQPs to these metalloids. However, the fact that TbAQP1 did not show this phenotype revealed differences in aquaglyceroporin structures.

Effect of pH on the metalloids uptake in Xenopus laevis oocytes expressing TbAQPs

Next we tried similar experiments in Xenopus laevis oocytes expressing TbAQPs at pH 5.5 and 7. As shown in Figure 4a, in contrast with the control cells, oocytes expressing TbAQPs were able to transport $\mathrm{Sb}$ (III) at both 5.5 and $7 \mathrm{pH}$ values, but the uptake at pH 5.5 was significantly reduced. This result is consistent with the previous observations with yeast transformants.

The effect of $\mathrm{pH}$ variations in the transport of As(III) shed different results for each TbAQP. For TbAQP3 the decrease in pH lowered the As(III) intake; for TbAQP2 a rather moderate though still statistically significant effect was registered; while for TbAQP1 virtually no effect was observed (Fig. 4b). These data are also consistent with the experiments in yeast. 
Fig. 4. As(III) and $\mathrm{Sb}(\mathrm{III})$ pH-dependent permeability in Xenopus laevis oocytes expressing TbAQPs. Oocytes were injected with water (control) or with $10 \mathrm{ng}$ of TbAQP1, 2 or 3 - cRNA. Upper panel represents $\mathrm{Sb}(\mathrm{III})$ uptake and lower panel denotes As(III) uptake. Graphics represent the mean \pm SD from at least three independents experiments. Statistical analysis was performed using GraphPad Prism software (GraphPad Software Inc, La Jolla, CA). Results with $\mathrm{p}<0.05$ were considered as statistically significant.

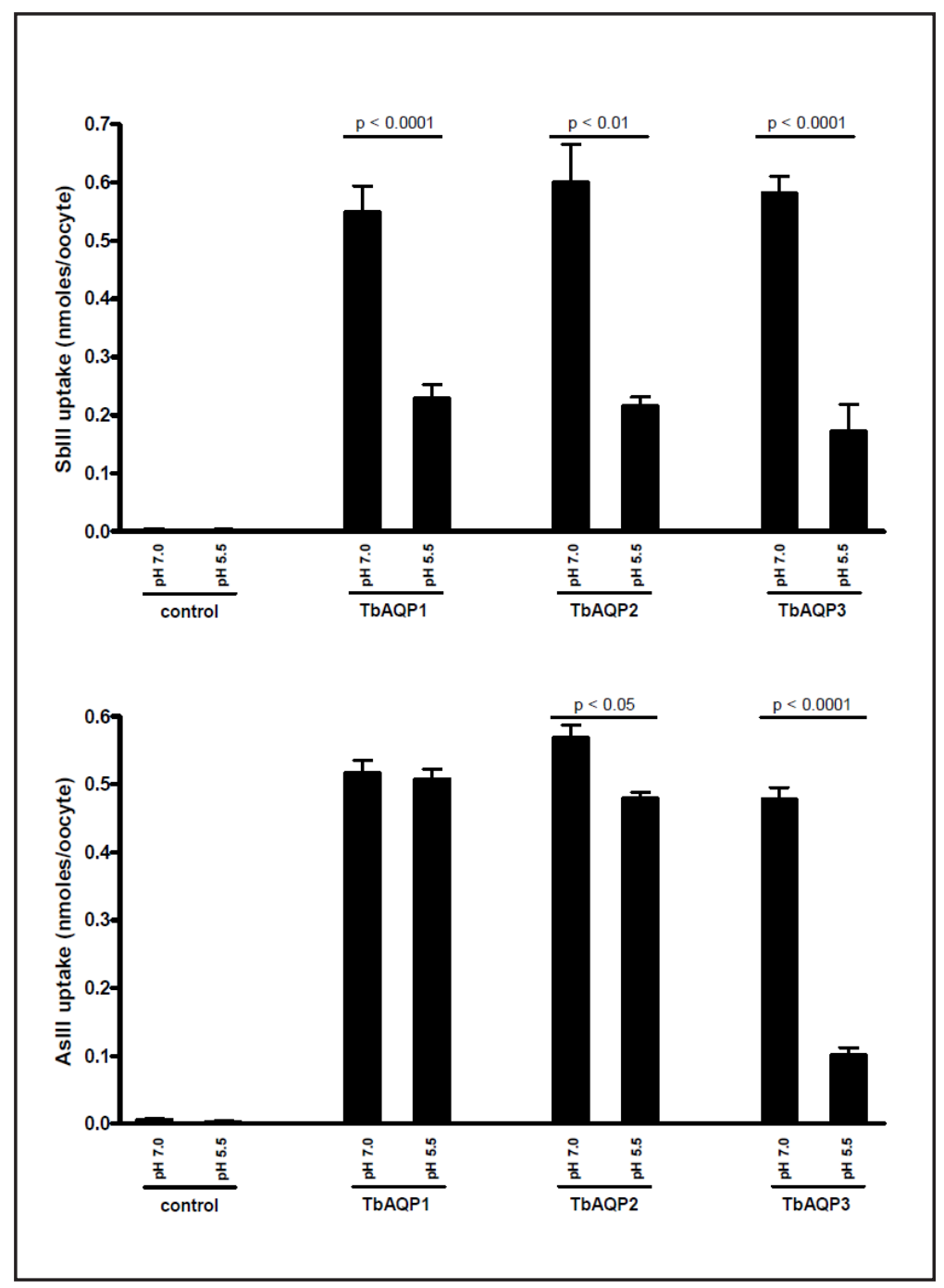

Table 1. $\mathrm{IC}_{50}$ values for the different metalloids in Trypanosoma brucei bloodstream forms

\begin{tabular}{lc}
\hline Compound & IC $_{50}$ in bloodstream $(\mu \mathrm{M})$ \\
\hline Arsenic V & $7.0 \pm 0.3$ \\
Arsenic III & $0.31 \pm 0.06$ \\
Antimony III & $0.103 \pm 0.01$ \\
\hline
\end{tabular}

Anti-proliferative effects of As(III), As(V) and Sb(III) on T. brucei

As(III) in the form of arsenic trioxide (Trisenox®), although very toxic and difficult to apply, is currently used against acute promyelocytic leukaemia being the most effective drug available against this disease [21]. With this in mind, we decided to evaluate As(III) cytotoxicity in trypanosomes. To this end, the $\mathrm{IC}_{50}$ of the three metalloids here studied were determined as described in Material and Methods in T.brucei cell cultures. As shown in Table $\mathrm{I}$, all arsenic and antimony compounds killed the parasite in the $\mathrm{nM}$ to $\mu \mathrm{M}$ range, the order of effectiveness being $\mathrm{Sb}(\mathrm{III})>\mathrm{As}(\mathrm{III})>\mathrm{As}(\mathrm{V})$. 


\section{Discussion}

The Trypanosoma brucei bloodstream form possesses three aquaglyceroporins namely: TbAQP1 located at the flagellum, TbAQP2 in the flagellar pocket and TbAQP3 in the plasma membrane $[7,13,22]$. Using RNAi technology, Bassarak et al. demonstrated that after hyposmotic shock these channels, in particular TbAQP2, participate in cell volume regulation. TbAQPs are also associated with drug uptake, as in the case of dihydroxyacetone, an innocuous compound for humans but cytotoxic for T. brucei which is very efficiently transported into the parasite [23, 24]. However, the ability of TbAQPs to uptake As(III) and $\mathrm{Sb}(\mathrm{III})$, which are basic components of several antiparasitic drugs against trypanosomatids, has not yet been tested. This fact, together with a recent report that in T. brucei TbAQP2 is associated with the toxic effects of the arsenical drug melarsoprol, [7], prompted us to investigate the trivalent arsenic transport in this parasite. Using two different protein expression systems, Saccharomyces cerevisiae and Xenopus laevis oocytes, we have shown that trypanosomal aquaglyceroporins are highly permeable for both trivalent arsenic and trivalent antimony. The heterologous expression of the three different T. brucei AQPs in yeast fps1 $\Delta$ mutant cells promoted the uptake of As(III) and Sb(III) through these channels increasing the sensitivity of these cells to these metalloids, and provoking cell death [13].

Since heterologous expression experiments using aquaglyceroporins from different organisms including mammals (AQP9) [25, 26], plants (nodulin-26-like intrinsic proteins (NIP)) [27] and yeast (Fps1p deleted of the N-terminal regulatory domain) [16] have shed similar results, our findings strongly suggest that TbAQPs are responsible for the uptake of these metalloids compounds.

We also performed direct transport assays in Xenopus laevis oocytes expressing TbAQPs, and the results of these experiments were consistent with the phenotypes observed in yeast, that is, $\mathrm{As}(\mathrm{III})$ and $\mathrm{Sb}(\mathrm{III})$ were efficiently transported by the three TbAQPs, with an average of a 76 fold increase in metalloid uptake with respect to control cells.

TbAQPs transport activity expressed in both yeast and Xenopus oocytes systems was sensitive to $\mathrm{pH}$ changes, in the case of $\mathrm{Sb}$ (III) a similar and significant effect of $\mathrm{pH}$ was observed for all three AQPs. In the case of As(III) however, TbAQP2 and 3 showed a pHdependent uptake, whereas TbAQP1 showed a pH insensitivity. Some explanations for these differences could be: A) the $\mathrm{Sb}$ (III) atomic weight is bigger than that of As(III) (122 and 75 , respectively), therefore these differences in molecular volumes may influence the permeability rate through TbAQPs; and B) the differences in uptake are due to variations in TbAQPs protein structure e.g., differences in some important amino acid positions among TbAQPs. However the fact that the three important regions for substrate permeability (i.e. the conserved NPA motif, the selectivity filter -ar/R region-, and the remaining pore-lining residues) are practically identical for the TbAQP1 and $3[13,25]$, gives little support for this option. Nonetheless, other amino acids, not directly involved in pore formation, can significantly modify the pore's permeability. This was recently demonstrated for two Killifish aquaglyceroporins KfAQP3a and KfAQP3b, that although sharing 96\% amino acid identity and showing no amino acid differences in the channel region, where the former does not allow the passage of As(III), while the later does. The only important structural discrepancy is located in the C-terminus, being Gly-Lys-Ser for kfAQP3a and Ala-Asn-Cys in kfAQP3b. When kfAQP3a C-terminal amino acid was changed to Ala-Asn-Cys by in vitro mutagenesis, the channel became permeable for trivalent arsenic [9]. There are other examples in the literature related to the connecting loops among AQP transmembrane domains, e.g. Glu125 in loop C of Plasmodium falciparum aquaglyceroporin (PfAQP) is responsible for a high capability of water transport that is largely abolished by changing this amino acid [28]. Likewise, mutation of Glu152 to Ala, within the same loop in Aquaglyceroporin 1 from Leishmania (LmAQP1), affected water as well as metalloid permeation, but not the glycerol transport [29]. These findings demonstrate that a fine-tuning of selectivity and capability for substrate transport are not exclusive of the classical three regions mentioned above, and that the three TbAQPs here studied present enough differences at their C-termini as well as 
in their $\mathrm{C}$ loops to account for the observed differences in permeability [13]. However, given the low degree of amino acid conservation among the members of the aquaporin family, it is difficult to make predictions of permeability behavior based on sequence comparisons. Therefore, additional experimental data are required to reveal the structural determinants of this pH-depending selectivity for As(III) and Sb(III) observed in the TbAQPs.

As(III) and Sb(III) show prominent effects on parasites, killing them at a low nM to $\mu \mathrm{M}$ range. In this regard the concentration of arsenic trioxide determined in plasma of Trisenox@-treated promyelocytic leukaemia patients is in the range of $300-2900 \mathrm{nM}$ [30, 31 , which is equal or tenfold higher than the $\mathrm{IC}_{50}$ values for this compound in T. brucei (Table 1). In addition Trisenox $®$ is not only detected in blood but also in cerebrospinal fluid [32]. Since the treatment of sleeping sickness is still problematic, and melarsoprol, one of the most prominent drugs used for late stage infections, has deadly side effects in about $5 \%$ of treated patients [33], the potential of Trisenox $®$ as an alternative drug to treat sleeping should be explored.

Taken together, we have found that T. brucei aquaglyceroporins can serve as entry routes for $\mathrm{As}(\mathrm{III})$ and $\mathrm{Sb}(\mathrm{III})$ into the parasitic cell, and that this uptake is $\mathrm{pH}$ sensitive. We also observed that TbAQPs are bi-directional channels for As(III). Finally, since TbAQP2 has been implicated in the drug resistance to the trypanocidal drugs melarsoprol and pentamidine $[7,8]$, and considering our results indicating the role of TbAQP in the uptake of As(III) drugs like Trisenox®, we believe these channels should be further studied in order to explore their potential as drug targets, drug delivery systems, and drug resistance cases in deadly infections such as sleeping sickness.

\section{Acknowledgements}

This work was supported by the Consejo de Desarrollo Científico y Humanístico from the Central University of Venezuela (PG 09.00.7059.2007 and PG 09-7059-2007/2), and the Venezuelan Ministry of Science, Technology and Innovation (Foundation IDEA, POA-2011, POA-2012). NLU and KF received a short-term fellowship from the Deutscher Akademischer Austausch Dienst (DAAD). UNU BIOLAC covered the publication fee. The authors would like to thank Ms. Sharon Sumpter for revising the English version of the manuscript.

\section{References}

1 Lepesheva GI, Waterman MR: Sterol 14alpha-Demethylase (CYP51) as a Therapeutic Target for Human Trypanosomiasis and Leishmaniasis. Curr Top Med Chem 2011;11:2060-2071.

2 Pereira CA, Silber AM: On the evolution of hexose transporters in kinetoplastids protozoans. Plos One 2012; 7:e36303.

-3 Baker N, de Koning H, Maser P, Horn D: Drug resistance in African trypanosomiasis: the melarsoprol and pentamidine story. Trends Parasitol 2013;29:110-118.

4 Bouteille B, Oukem O, Bisser S, Dumas M: Treatment perspectives for human African trypanosomiasis. Fundam Clin Pharmacol 2003;17:171-181.

5 Gourbal B, Sonuc N, Bhattacharjee H, Legare D, Sundar S, Ouellette M, Rosen BP, Mukhopadhyay R: Drug uptake and modulation of drug resistance in Leishmania by an aquaglyceroporin. J Biol Chem 2004;279:31010-31017.

6 Marquis N, Gourbal B, Rosen BP, Mukhopadhyay R, Ouellette M: Modulation in aquaglyceroporin AQP1 gene transcript levels in drug-resistant Leishmania. Mol Microbiol 2005;57:1690-1699.

7 Baker N, Glover L, Munday J, Aguinaga-Andrés D, Barrett MP, de Koning HP, Horn D: Aquaglyceroporin 2 controls susceptibility to melarsoprol and pentamidine in African trypanosomes. Proc Natl Acad Sci USA 2012;109:10996-11001.

8 Alsford S, Eckert S, Baker N, Glover L, Sanchez-Flores A, Leung KF, Turner DJ, Field MC, Berriman M, Horn D: High-throughput decoding of antitrypanosomaldrug efficacy and resistance. Nature 2012;482:232-236.

-9 Jung D, MacIver B, Jackson JB, Barnaby R, Sato JD, Zeidel ML, Shaw JR, Stanton BA: A novel aquaporin 3 in killifish (Fundulus heteroclitus) is not an arsenic channel. Toxicol Sci 2012;127:101-109. 
Uzcátegui et al.: Uptake of Arsenite and Antimonite by TbAQPs

10 Ausubel F, Brent R, Kingston R, Moore D, Seidman J, Struhl K: Current Protocol in Molecular Biology: John Wiley \& Sons, New York, 2000, pp13.1.2-13.1.7.

11 Wagner CA, Friedrich B, Setiawan I, Lang F, Bröer S: The use of Xenopus laevis oocytes for the functional characterization of heterologously expressed membrane proteins. Cell Physiol Biochem 2000;10:1-12.

12 Hamm B, Schindler A, Mecke D, Duszenko M: Differentiation of Trypanosoma brucei bloodstream trypomastigotes from long slender to short stumpy- like forms in axenic culture. Mol Biochem Parasitol 1990;40:13-22.

13 Uzcategui NL, Szallies A, Pavlovic-Djuranovic S, Palmada M, Figarella K, Boehmer C, Lang F, Beitz E, Duszenko M: Cloning, heterologous expression, and characterization of three aquaglyceroporins from Trypanosoma brucei. J Biol Chem 2004;279:42669-42676.

14 Figarella K, Uzcategui NL, Zhou Y, LeFurgey A, Ouellette, M, Bhattacharjee H, Mukhopadhyay R: Biochemical characterization of Leishmania major aquaglyceroporin LmAQP1: possible role in volume regulation and osmotaxis. Mol Microbiol 2007;65:1006-1017.

15 15. Bodley AL, McGarry MW, Shapiro TA: Drug cytotoxicity assay for african trypanosomes and Leishmania species., J Infect Dis 1995;172:1157-1159.

16 16. Wysocki R, Chery CC, Wawrzycka D, Van Hulle M, Cornelis R, Thevelein JM, Tamás MJ.: The glycerol channel Fps1p mediates the uptake of arseniteand antimonite in Saccharomyces cerevisiae. Mol Microbiol 2001;40:1391-1401.

17 Bienert GP, Thorsen M, Schüssler MD: A subgroup of plant aquaporins facilitate the bi-directional diffusionof $\mathrm{As}(\mathrm{OH}) 3$ and $\mathrm{Sb}(\mathrm{OH}) 3$ across membranes. BMC Biol 2008;6:1-15.

18 Rosen BP: Biochemistry of arsenic detoxication. FEBS Lett 2002;529:86-92.

19 Maciaszczyk-Dziubinska E, Migdal I, Migocka M, Bocer T, Wysocki R: The yeast aquaglyceroporin Fps1p is a bidirectional arsenite channel. FEBS Lett 2010;584:726-732.

20 Ramírez-Solís A, Mukopadhyay R, Rosen B, Stemmler TL: Experimental and theoretical characterization of arsenite in water: Insights into the coordination environment of As-0. Inorg Chem 2004;43:2954-2959.

-21 Breccia M, Lo-Coco F: Arsenic trioxide for management of acute promyelocytic leukemia: current evidence on its role in front-line therapy and recurrent disease. Expert Opin Pharmacother 2012;13:1031-1043.

22 Bassarak B, Uzcátegui NL, Schönfeld C, Duszenko M: Functional characterization of three aquaglyceroporins from Trypanosoma brucei in osmoregulation and glycerol transport. Cell Physiol Biochem 2011;27:411-420.

23 Uzcategui NL, Carmona-Gutierrez D, Denninger V, Schoenfeld C, Lang F, Figarella K, Duszenko M: Antiproliferative Effect of Dihydroxyacetone on Trypanosoma brucei Bloodstream Forms: Cell Cycle Progression, SubcellularAlterations, and Cell Death. Antimicrob Agents Chemother 2007;51:3960-3968.

24 Uzcátegui NL, Denninger V, Merkel P, Schoenfeld C, Figarella K, Duszenko M: Dihydroxyacetone induced autophagy in african trypanosomes. Autophagy 2007;3:626-629.

25 Wu B, Beitz E: Aquaporins with selectivity for unconventional permeants. Cell Mol Life Sci 2007;64:24132421.

26 Liu Z, Carbrey JM, Agre P, Rosen BP: Arsenic trioxide uptake by human and rat aquaglyceroporins. Biochem Biophys Res Commun 2004;316:1178-1185.

27 Bhattacharjee H, Mukhopadhyay R, Thiyagarajan S, Rosen BP: Aquaglyceroporins: ancient channels for metalloids. J Biol 2008;7:33.

28 Beitz E, Pavlovic-Djuranovic S, Yasui M, Agre P, Schultz JE: Molecular dissection of water and glycerolpermeability of the aquaglyceroporin from Plasmodium falciparum by mutational analysis. Proc Natl Acad Sci USA 2004;101:1153-1158.

29 Uzcategui NL, Zhou Y, Figarella K, Ye J, Mukhopadhyay R, Bhattacharjee H: Alteration in glycerol and metalloid permeability by a singlemutation in the extracellular C-loop of Leishmania major aquaglyceroporin LmAQP1. Mol Microbiol 2008;70:1477-1486.

-30 Soignet SL, Maslak P, Wang ZG, Jhanwar S, Calleja E, Dardashti LJ, Corso D, DeBlasio A, Gabrilove J, Scheinberg DA, Pandolfi PP, Warrell RP Jr: Complete remission after treatment of acute promyelocytic leukemia with arsenic trioxide. N Engl J Med 1998;339:1341-1348.

-31 List A, Beran M, DiPersio J, Slack J, Vey N, Rosenfeld CS, Greenberg P: Opportunities for Trisenox (arsenic trioxide) in the treatment of myelodysplastic syndromes. Leukemia 2003;17:1499-1507.

-32 Helwig A, Klemm M, Schüttig R, Röllig C, Wassilew N, Ehninger G, Illmer T: Arsenic-induced APL differentiation in cerebrospinal fluid. Leuk Res 2007;31:703-705.

33 Burri C: An alternative form of melarsoprol in sleeping sickness: is an old drug always the best basis for a new one? Trends Parasitol 2012;28:354-355. 